\title{
Study on Poly(3,4-ethylenedioxythiophene) Thin Film Vapour Phase-Polymerized with Iron(III)Tosylate on AcOH-Catalyzed 3-Aminopropyltriethoxysilane Self-Assembled Monolayer
}

\author{
Sangil Choi, Wondae Kim, and Sungsoo Kim ${ }^{\dagger}$
}

\begin{abstract}
In this study, PEDOT thin films polymerized with Iron(III)tosylate (Fe(PTS) $)_{3}$ and grown on acetic acid-catalyzed 3aminopropyltriethoxysilane self-assembled monolayer (APS-SAM) surfaces by VPP method have been investigated. PEDOT thin films were synthesized on APS self-assembled $\mathrm{SiO}_{2}$ wafer surface at two different concentrations $(20 \mathrm{wt} \%$ and $40 \mathrm{wt} \%$ ) and growth time ( 3 and 30 minutes), and then they were compared. PEDOT vapour phase-polymerized with $40 \mathrm{wt} \% \mathrm{Fe}(\mathrm{PTS})_{3}$ oxidant completely formed a thin film on acetic acid-catalyzed APS-SAM surface while with $20 \mathrm{wt} \%$ $\mathrm{Fe}(\mathrm{PTS})_{3}$ did not at all. It means that the oxidant can be uniformly coated on acetic acid-catalyzed APS-SAM surface at the $40 \mathrm{wt} \%$ concentration, which gives rise to the uniform growth of PEDOT thin film on it.
\end{abstract}

Key words: Poly(3,4-ethylenedioxythiophene), 3-Aminopropyltriethoxysilane, Vapor Phase Polymerization, Iron(III)tosylate

\section{Introduction}

For decades, conductive $\pi$-conjugated polymers have fascinated many scientists with their great potential for applicability to the next-generation flexible display device such as organic thin film transistors (OTFTs) and organic light emitting diodes (OLEDs) ${ }^{[1]}$. Among these polymers, poly(3,4-ethylenedioxythiophene) (PEDOT) has been particularly focused on as a potential component of OTFT or OLED devices due to its superb material characteristics ${ }^{[2]}$. PEDOT is electrochemically very stable, and it has a low bandgap energy $(1.5 \sim 1.7 \mathrm{eV})$ and a considerably high electrical conductivity. PEDOT thin film is generally synthesized via two different methods, i.e., spin-coating or oxidative vapour phase polymerization (VPP). Electrical characteristics of the thin film prepared by those methods are critically affected by its growth morphology. In particular, our recent study showed that the morphology of PEDOT thin film polymerized with $\mathrm{FeCl}_{3}$ and grown on a high quality 3-amonopropyltriethoxythiophene self-assem-

${ }^{1}$ Department of Nano-Polymer Materials Engineering,Pai Chai University, Daejeon, 302-735

${ }^{\dagger}$ Corresponding author : skim@pcu.ac.kr

(Received: December 10, 2012, Revised : December 15, 2012, Accepted : December 21, 2012) bled monolayer (APS-SAM) is noticeably different from that of the thin film grown on a relatively low quality APS-SAM ${ }^{[3]}$. The film grown on the high quality SAM by vapour phase polymerization (VPP) method is denser (or less porous) and more robustly adhered to silicon oxide surface. It suggests that APS-SAM helps to improve the PEDOT film quality, thereby improving the electrical characteristics of the film. It means that the film morphology is considerably affected by APSSAM, itself as well as its quality. Therefore, in this study, in order to improve electrical characteristics of PEDOT thin film vapour phase-polymerized and then doped with $\mathrm{Fe}$ (para-toluenesulfonate $)_{3}\left(\mathrm{Fe}(\mathrm{PTS})_{3}\right)$, the thin films were grown on two differently prepared (both high and low quality) APS-SAM, and they were compared in terms of oxidant concentration and growth time.

\section{Experimental Section}

Chemicals used in this experiment, iron(III)tosylate (or Iron (III) para-tolenesulfonate), 3-aminopropyltirethoxysilane (APS), 3,4-ethylenedioxythiophene, were purchased from Sigma/Aldrich company, USA, and toluene (reagent grade) was refluxed under $\mathrm{Na}$ metal and further distilled under $\mathrm{N}_{2}$. Oxidized silicon wafer 


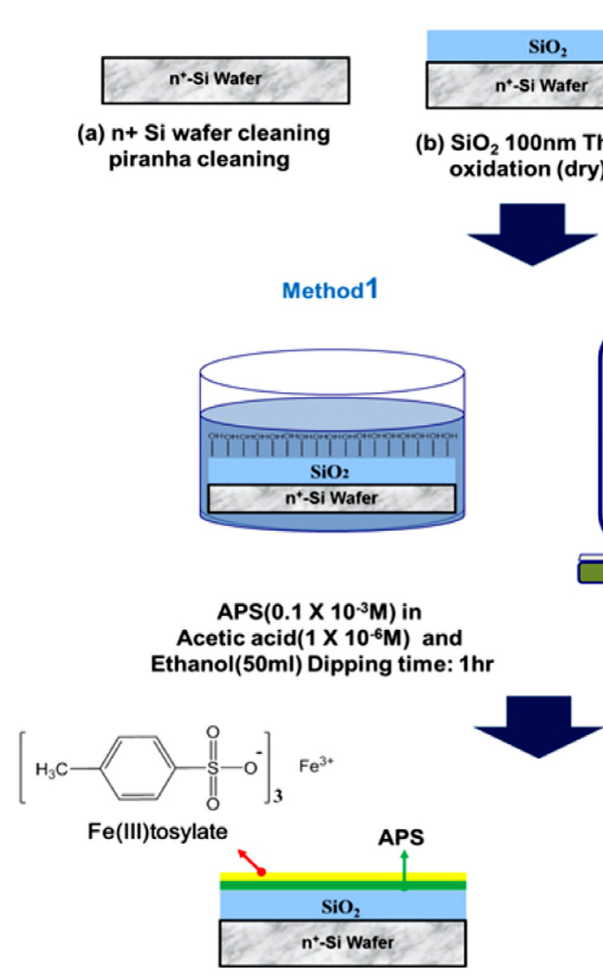

(d) Fe(III)tosylate spin coating

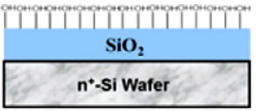

(c) RCA cleaning process hydroxylation

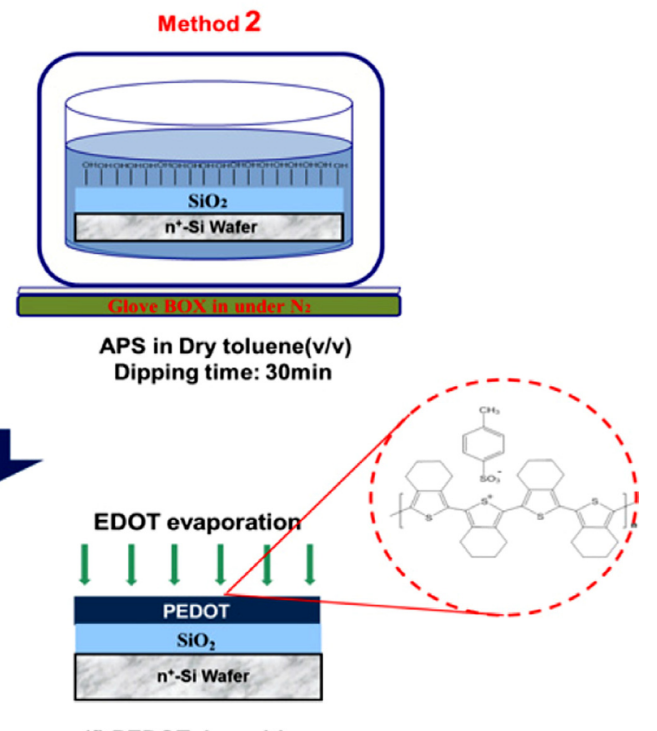

(f) PEDOT deposition

Fig. 1. Schematic diagram of PEDOT vapour phase polymerization (VPP) method. Fe(PTS) 3 was used as a dopant as well as an oxidant for the VPP process.

$(1.5 \mathrm{~cm} \times 1.5 \mathrm{~cm})$ was ultra-sonicated in ethanol for $30 \mathrm{~min}$. after ethanol wash. And then, the wafer was repeatedly treated with piranha solution and DI water to remove organic/inorganic contaminants on it. After that, to remove water molecules adsorbed on the wafer surface, it was dried in a vacuum oven at $70^{\circ} \mathrm{C}$ for $30 \mathrm{~min}$. On the cleaned wafer, two different samples of APS monolayer were self-assembled at completely different conditions. First sample is a monolayer prepared in a glove box under a strictly controlled humidity $(<10$ $\% \mathrm{RH})$. And also completely anhydrous toluene was used as a solvent throughout the preparation. The wafer substrate was dipped into APS $(120 \mu \mathrm{L})$ solution dissolved in $60 \mathrm{~mL}$ of anhydrous toluene for $30 \mathrm{~min}$. Second one is a APS monolayer self-assembled in atmospheric environment without any suppression of humidity. The monolayer was self-assembled in APS $\left(0.1 \times 10^{-3}\right.$ M) solution mixed with acetic acid $\left(1 \times 10^{-6} \mathrm{M}\right)$ in $50 \mathrm{~mL}$ of ethanol for $1 \mathrm{~h}$. Two different oxidant solutions for the growth of PEDOT thin film were prepared with
$20 \mathrm{wt} \%$ and $40 \mathrm{wt} \%$ iron(III)tosylate $6 \mathrm{H}_{2} \mathrm{O}$ dissolved in ethanol. Oxidant films were spin-coated on the wafer substrates at $2500 \mathrm{rpm}$ for $90 \mathrm{~s}$. And then, as shown in Fig. 1, PEDOT was vapor phase-polymerized in an oven at $70^{\circ} \mathrm{C}$ for $30 \mathrm{~min}$. In order to characterize APSSAM and PEDOT thin film, AFM (VEECO, Nanoman, U.S.A) and FE-SEM (FEI, Sirion model, Netherlands) were used, and sheet resistance of PEDOT thin film were measured using a 4-point probe (A.I.T, CMTSR2000).

\section{Results and Discussion}

Our previous study showed that the growth morphology and interfacial adhesion of PEDOT thin film is highly dependent upon APS-SAM ${ }^{[3]}$. That is, APSSAM helps $\mathrm{FeCl}_{3}$ solution to uniformly coat $\mathrm{SiO}_{2}$ surface and also to promote adhesion between PEDOT thin film and $\mathrm{Si}$ wafer substrate, thereby resulting in improved film quality. PEDOT thin film is vapour 


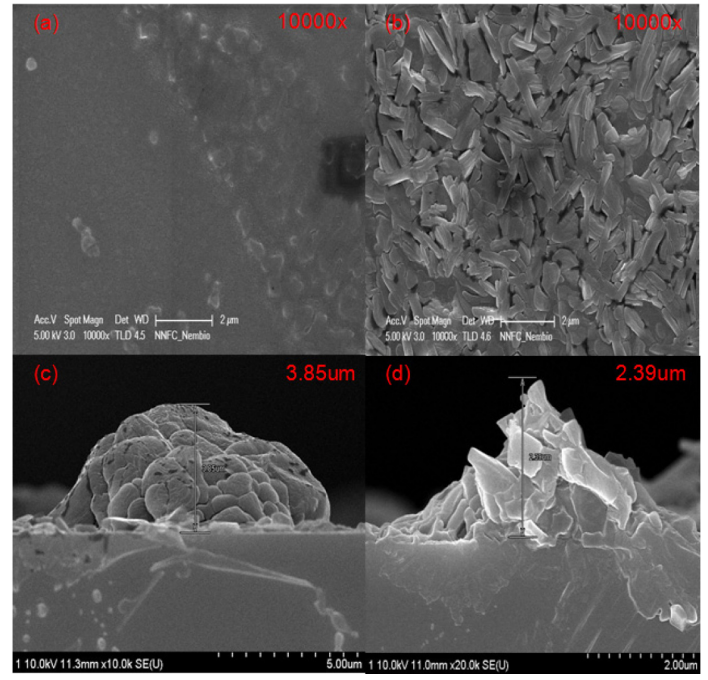

Fig. 2. FE-SEM images of PEDOT thin films grown on acetic acid-catalyzed APS-SAMs. All of the films used $20 \mathrm{wt} \% \mathrm{Fe}(\mathrm{PTS})_{3}$. The films in (a) and (c) images were grown for $3 \mathrm{~min}$ and those in (b) and (d) images for $30 \mathrm{~min}$.

phase-polymerized generally via two different oxidants, i.e., $\mathrm{FeCl}_{3}$ and $\mathrm{Fe}(\mathrm{PTS})_{3}$. Both oxidant have their own merits. However, unlike $\mathrm{FeCl}_{3}, \mathrm{Fe}(\mathrm{PTS})_{3}$ is more advantageous to the deposition of less porous film. Thus, as the first section of these serial publications, PEDOT thin films polymerized with the $\mathrm{Fe}(\mathrm{PTS})_{3}$ oxidant and grown on acetic acid-catalyzed APS-SAMs by VPP method have been mainly investigated. Fig. 2 shows FE-SEM images of PEDOT thin films grown on acetic acid catalyzed APS-SAM surface via only $20 \mathrm{wt} \% \mathrm{Fe}(\mathrm{PTS})_{3}$. In general, it has been known that $40 \mathrm{wt} \% \mathrm{Fe}(\mathrm{PTS})_{3}$ is used for the synthesis of highly conductive VPPPEDOT ${ }^{[4]}$ However, such concentration is too high for the oxidant to over-dominate the physico-chemical properties of the polymer, itself. Therefore, this study tried to reduce the concentration of $\mathrm{Fe}(\mathrm{PTS})_{3}$ to the half (20 wt $\%$ ) of currently known optimal one when a base inhibitor is used for the VPP reaction. ${ }^{[4]}$ However, as shown in Fig. 2, PEDOT didn't form films at both 3 and 30 minutes of growth time. It may suggest that the oxidant solution was not uniformly coated on the acetic acid-catalyzed and thus very rough APS-SAM surface, which is a poor quality ${ }^{[3]}$. Thereby, it resulted in such incompletely formed PEDOT films. Or more convincingly, less amount of the oxidant and rough and quite hydorphobic APS monolayer surface, they both play

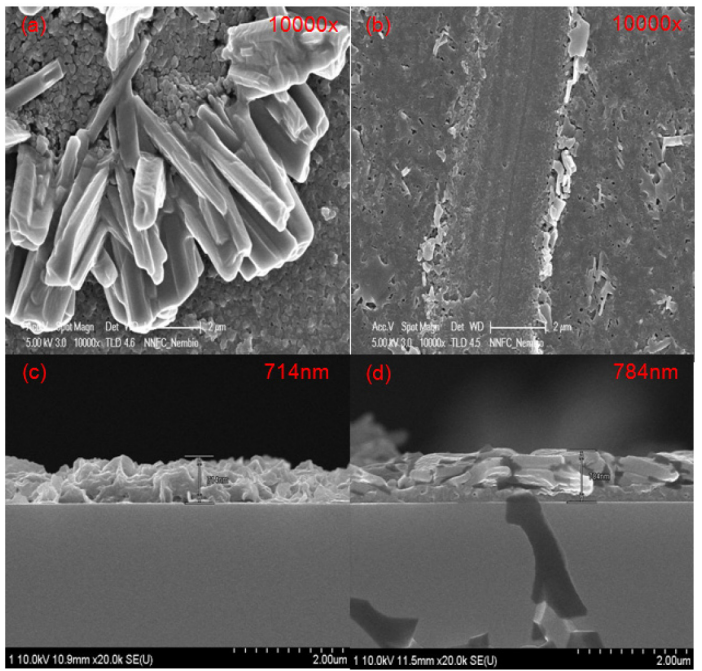

Fig. 3. FE-SEM images of PEDOT thin films grown on acetic acid-catalyzed APS-SAMs. All of the films used $40 \mathrm{wt} \% \mathrm{Fe}(\mathrm{PTS})_{3}$. The films in (a) and (c) images were grown for 3 minutes and those in (b) and (d) images for 30 minutes.

important roles at the same time in very poorly formed PEDOT films. Indeed, FE-SEM images shown in Fig. 3 , clearly support the latter conjecture. In other words, VPP-PEDOT on $40 \mathrm{wt} \% \mathrm{Fe}(\mathrm{PTS})_{3}$-coated $\mathrm{SiO}_{2}$ surface at two different growth time (3 and 30 minutes) completely formed films. It means that the $40 \mathrm{wt} \%$ oxidant solution is uniformly coated on acetic acid-catalyzed APS-SAM surface. As a result, PEDOT also uniformly grows on the oxidant-coated surface even at the relatively short growth time of 3 minutes as shown in Fig. 3c. In addition, FE-SEM images in both Fig. 2 and Fig. 3 indicate that PEDOT grows faster on $\mathrm{Fe}(\mathrm{PTS})_{3}$-coated APS-SAM surface than on the same oxidant-coated bare $\mathrm{SiO}_{2}$ surface. It is clearly supported by the fact of which PEDOT thin film was nearly completely formed even at very short growth time of 3 minutes. Such growth time is as short as that of the PEDOT thin film polymerized with only $3 \mathrm{wt} \% \mathrm{FeCl}_{3}$ even if the $\mathrm{FeCl}_{3}$ doped film grows usually much faster than $\mathrm{Fe}(\mathrm{PTS})_{3}$ doped one does ${ }^{[3,4]}$. More detail kinetic and crystallographic studies are underway.

\section{Conclusion}

This study demonstrated that PEDOT vapour phase- 
polymerized with $40 \mathrm{wt} \% \mathrm{Fe}(\mathrm{PTS})_{3}$ oxidant completely forms a thin film on acetic acid-catalyzed APS-SAM surface while PEDOT polymerized with $20 \mathrm{wt} \%$ oxidant does not at all. It means that the oxidant can be uniformly coated on acetic acid-catalyzed APS-SAM surface at the $40 \mathrm{wt} \%$ concentration, which gives rise to the uniform growth of PEDOT thin film on it.

\section{Acknowledgment}

This study was financially supported by Flexolution Corporation, Yongin, Korea.

\section{References}

[1] K. Kaneto, M. Maxfield, D. P. Nairns, A. G. MacDiarmid, and A. J. Heeger, "Electrochemistry of polyacetylene, $(\mathrm{CH}) \mathrm{x}$, characteristics of polyacetylene cathodes", J. Chem. Soc., Faraday Trans. 1, Vol. 78, pp. 3417-3429, 1982.
[2] G. Gustafsson, Y. Cao, G. M. Tracy, F. Klavetter, N. Colaneri, and A. J. Heeger, "Flexible light-emitting diodes made from soluble conducting polymers", Nature, Vol. 357, pp. 477-479, 1993.

[3] S. Choi, W. Kim, and S. Kim, "Development of highly conductive poly(3,4-ethylenedioxythiophene) thin film using high quality 3-aminopropyltriethoxysilane self-assembled monolayer", J. Chosun Natural Sci., Vol. 4, pp. 294-297, 2011.

[4] B. Winther-Jensen, D. W. Breiby, and K West, "Base inhibited oxidative polymerization of 3,4-ethylenedioxythiophene with iron(III)tosylate", Synth. Metals, Vol. 152, pp. 1-4, 2005. 